\title{
Homoclinic Trajectories of Non-Autonomous Maps
}

\author{
Thorsten Hüls* \\ Fakultät für Mathematik, Universität Bielefeld \\ Postfach 100131, 33501 Bielefeld, Germany \\ huels@math.uni-bielefeld.de
}

March 20, 2009

\begin{abstract}
For non-autonomous difference equations of the form

$$
x_{n+1}=f\left(x_{n}, \lambda_{n}\right), \quad n \in \mathbb{Z}
$$

we consider homoclinic trajectories. These are pairs of trajectories that converge in both time directions towards each other. Assuming hyperbolicity, we derive a numerical method to compute homoclinic trajectories in two steps. In the first step one trajectory is approximated by the solution of a boundary value problem and precise error estimates are given. In particular, influences of parameters $\lambda_{n}$ with $|n|$ large are discussed in detail. A second trajectory that is homoclinic to the first one is computed in a subsequent step as follows. We transform the original system into a topologically equivalent form having zero as an $n$-independent fixed point. Applying the boundary value ansatz to the transformed system, we obtain a non-autonomous homoclinic orbit, converging towards the origin, cf. Hüls (2006). Transforming back to the original coordinates leads to the desired homoclinic trajectories. The numerical method and the validity of the error estimates are illustrated by examples.
\end{abstract}

Keywords: Non-autonomous discrete time dynamical systems, Homoclinic trajectories, Numerical approximation, Error analysis.

*Supported by CRC 701 'Spectral Structures and Topological Methods in Mathematics'. 


\section{Introduction}

For autonomous systems it is well known that the dynamics in a neighborhood of a homoclinic orbit is chaotic, see Smale (1967). In subsequent years, homoclinic orbits were analyzed in various studies, cf. Palis \& Takens (1993) for an historical overview. Of particular importance are approximation results, see for example Beyn (1990) for continuous time systems as well as the current version of the bifurcation toolbox Matcont Dhooge et al. (2003), Ghaziani et al. (2009) for an implementation. For discrete time systems, we refer to Beyn \& Hüls (2004), Beyn et al. (2004), Beyn \& Kleinkauf (1997), Hüls (2005). The opposite question, whether pseudo orbits lead to exact orbits - so called shadowing results - are discussed in detail in Pilyugin (1999). Shadowing techniques for homoclinic and heteroclinic orbits, converging towards periodic orbits, in discrete and continuous time, are developed in Coomes et al. (2005) and Coomes et al. (2007).

In several realistic applications from physics or mathematical biology, the limitation to autonomous systems is too restrictive. A typical example is a population model, where the carrying capacity of the environment varies in time, cf. Elaydi \& Sacker $(2005 a, b)$, Beyn et al. (2008). These models require the development of non-autonomous tools. In Hüls (2006) non-autonomous difference equations of the form

$$
x_{n+1}=f_{n}\left(x_{n}\right), \quad n \in \mathbb{Z}
$$

are considered. It is assumed that $f_{n} \in \mathcal{C}^{\infty}\left(\mathbb{R}^{k}, \mathbb{R}^{k}\right)$ are diffeomorphisms for all $n \in \mathbb{Z}$, having zero as an $n$-independent fixed point, i.e. $f_{n}(0)=0$ for all $n \in \mathbb{Z}$. With respect to this fixed point, a homoclinic orbit is computed numerically in Hüls (2006). Note that the points of a homoclinic orbit lie in the intersection of the corresponding stable and unstable fiber bundles of the fixed point 0 . These fiber bundles are the non-autonomous equivalent of the invariant manifolds in autonomous systems, cf. Hirsch et al. (1977), Pötzsche \& Siegmund (2004).

More precisely, a homoclinic orbit $\bar{x}_{\mathbb{Z}}=\left(\bar{x}_{n}\right)_{n \in \mathbb{Z}}$ w.r.t. the fixed point 0 is a solution of (1), fulfilling $\lim _{n \rightarrow \pm \infty} \bar{x}_{n}=0$. The proposed method for computing a finite approximation on some interval $J=\left[n_{-}, n_{+}\right] \cap \mathbb{Z}$, requires solving the boundary value problem

$$
0_{J}=\Gamma_{J}\left(y_{J}\right):=\left(\left(y_{n+1}-f_{n}\left(y_{n}\right)\right)_{n=n_{-}, \ldots, n_{+}-1}, b\left(y_{n_{-}}, y_{n_{+}}\right)\right),
$$

with an appropriately chosen boundary operator $b \in \mathcal{C}^{1}\left(\mathbb{R}^{2 k}, \mathbb{R}^{k}\right)$. The simplest choice are periodic boundary conditions $b\left(y_{n_{-}}, y_{n_{+}}\right)=y_{n_{-}}-y_{n_{+}}$. Under reasonable assumptions, the boundary value problem (2) possesses a unique solution in a sufficiently small neighborhood of the exact solution, cf. Hüls (2006).

In this paper, we push these ideas one step further by skipping the assumption that an $n$-independent fixed point exists. Then, the only candidate for the role of the fixed point $\bar{\xi}=0$ from the previous setup, is a bounded trajectory $\bar{\xi}_{\mathbb{Z}}$ of (1), cf. 
Langa et al. (2002). Thus a homoclinic orbit $\bar{x}_{\mathbb{Z}}$ is a trajectory, converging in both time directions towards $\bar{\xi}_{\mathbb{Z}}$, i.e.

$$
\lim _{n \rightarrow \pm \infty}\left\|\bar{x}_{n}-\bar{\xi}_{n}\right\|=0
$$

On the other hand $\bar{\xi}_{\mathbb{Z}}$ is also a homoclinic orbit w.r.t. $\bar{x}_{\mathbb{Z}}$. Due to this symmetry, we call two trajectories homoclinic if they satisfy (3).

Systems of the form (1) are typically generated by parameter dependent maps, where the parameter varies in time. We considered in this paper non-autonomous systems of the form

$$
x_{n+1}=f\left(x_{n}, \lambda_{n}\right), \quad n \in \mathbb{Z},
$$

where $\lambda_{\mathbb{Z}}$ denotes some sequence of parameter values, and analyze the following problems:

(1) Determine a bounded solution $\bar{\xi}_{\mathbb{Z}}$ of (4), given the sequence $\bar{\lambda}_{\mathbb{Z}}$.

(2) Determine an orbit $\bar{x}_{\mathbb{Z}}$, homoclinic to $\bar{\xi}_{\mathbb{Z}}$.

Note that both trajectories are generally not known explicitly.

In Section 2, we first introduce our basic assumptions and prove dichotomy results for the variational equation. Then we derive an algorithm for the numerical approximation of the bounded trajectory $\bar{\xi}_{\mathbb{Z}}$. For the computations, we solve the boundary value problem $\Gamma_{J}\left(\xi_{J}\right)=0$ on some interval $J=\left[n_{-}, n_{+}\right]$, using periodic boundary conditions. Doing so, the error at the outer points $\xi_{n_{ \pm}}$is quite large, since the boundary condition is not accurate and in addition each point $\bar{\xi}_{n}$ of the exact orbit depends on all parameter values $\bar{\lambda}_{\mathbb{Z}}$, cf. Figure 1 . Nevertheless, finite computations are justified, since errors at the boundary decrease exponentially fast toward the middle of the interval, see Theorem 5. By taking only the inner points, cf. Theorem 8, we obtain an approximation that is accurate up to any given accuracy.

In Section 3, an algorithm for computing a second trajectory $\bar{x}_{\mathbb{Z}}$ that is homoclinic to $\bar{\xi}_{\mathbb{Z}}$, is introduced. The idea is to consider the topologically equivalent system

$$
y_{n+1}=f\left(y_{n}+\bar{\xi}_{n}, \bar{\lambda}_{n}\right)-\bar{\xi}_{n+1}, \quad n \in \mathbb{Z}
$$

and apply the same boundary value approach, described above for getting a homoclinic orbit w.r.t. the fixed point 0, see also Hüls (2006). Transforming back to the original coordinates, we finally obtain an approximation of the homoclinic trajectory $\bar{x}_{\mathbb{Z}}$.

For an illustration, we first consider Hénon's map in Section 4. One of its parameters is chosen at random and we get a non-autonomous system of the form (4). We indicate that the approach gives high accuracy approximations of bounded trajectories. In a second step, homoclinic trajectories are computed numerically.

The second example, a predator-prey model, is taken from mathematical biology, cf. Beddington et al. (1975), Murray (2002). The carrying capacity of the 
environment is a parameter that fluctuates in time and generates in this way a nonautonomous system of the form (4). For different amplitudes of the fluctuation, homoclinic trajectories are computed.

We finally note that non-autonomous homoclinic and heteroclinic trajectories also arise in the analysis of controllability in control problems of the form

$$
x_{n+1}=f\left(x_{n}, u_{n}\right), \quad n \in \mathbb{Z}, \quad u_{n} \text { control at time } n,
$$

where $f$ models, for example, roll motions of a ship, cf. Colonius et al. (2009).

\section{Bounded trajectories}

We start this section by introducing basic notions and assumptions. Throughout this paper, the non-autonomous system (1) is assumed to be generated by a parameter dependent map as in (4).

We impose the following assumptions on $f$.

A1 $f \in \mathcal{C}^{\infty}\left(\mathbb{R}^{k} \times \mathbb{R}, \mathbb{R}^{k}\right)$ and $f(\cdot, \lambda)$ is a diffeomorphism for all $\lambda \in \mathbb{R}$.

A2 There exists a bounded sequence $\bar{\lambda}_{\mathbb{Z}} \in \mathbb{R}^{\mathbb{Z}}$ such that (5) possesses the bounded solution $\bar{\xi}_{\mathbb{Z}}$.

A3 The variational equation

$$
u_{n+1}=D_{x} f\left(\bar{\xi}_{n}, \bar{\lambda}_{n}\right) u_{n}, \quad n \in \mathbb{Z}
$$

possesses an exponential dichotomy on $\mathbb{Z}$, cf. Appendix A, Definition 13.

Definition 1 For a given parameter sequence $\bar{\lambda}_{\mathbb{Z}}$, let $\bar{\xi}_{\mathbb{Z}}$ and $\bar{x}_{\mathbb{Z}}$ be two solutions of the non-autonomous difference equation

$$
x_{n+1}=f\left(x_{n}, \bar{\lambda}_{n}\right), \quad n \in \mathbb{Z} .
$$

The trajectories $\bar{\xi}_{\mathbb{Z}}$ and $\bar{x}_{\mathbb{Z}}$ are homoclinic to each other, if

$$
\lim _{n \rightarrow \pm \infty}\left\|\bar{x}_{n}-\bar{\xi}_{n}\right\|=0
$$

Let $J=\left[n_{-}, n_{+}\right] \cap \mathbb{Z}$ be a discrete interval, where the cases $n_{-}=-\infty$ and $n_{+}=\infty$ are included. We define the space of bounded sequences on $J$

$$
X_{J}:=\left\{u_{J}=\left(u_{n}\right)_{n \in J} \in\left(\mathbb{R}^{k}\right)^{J}: \sup _{n \in J}\left\|u_{n}\right\|<\infty\right\}
$$

equipped with the $\ell_{\infty}$-norm, and denote by $0_{J}$ the zero element in $X_{J}$. 
For a given sequence $\lambda_{\mathbb{Z}}$, an orbit $x_{\mathbb{Z}} \in X_{\mathbb{Z}}$, i.e. a solution of (5), is a zero of the operator $\Gamma: X_{\mathbb{Z}} \times \mathbb{R}^{\mathbb{Z}} \rightarrow X_{\mathbb{Z}}$, defined as

$$
\Gamma\left(\xi_{\mathbb{Z}}, \lambda_{\mathbb{Z}}\right):=\left(\xi_{n+1}-f\left(\xi_{n}, \lambda_{n}\right)\right)_{n \in \mathbb{Z}}
$$

Let $\bar{\lambda}_{\mathbb{Z}}$ be the sequence from assumption A2. In general, the bounded solution $\bar{\xi}_{\mathbb{Z}}$ is not known explicitly. Even worse, the sequence $\bar{\xi}_{\mathbb{Z}}$ is not convergent. The main task, we consider in this section, is to compute an approximation of this bounded trajectory. First, we prove that a bounded trajectory also exists in some neighborhood of $\bar{\xi}_{\mathbb{Z}}$, if the parameter sequence $\lambda_{\mathbb{Z}}$ varies slightly around $\bar{\lambda}_{\mathbb{Z}}$.

Lemma 2 Assume $\boldsymbol{A} 1-\boldsymbol{A}$ 3. Then there exist two neighborhoods $U\left(\bar{\lambda}_{\mathbb{Z}}\right)$ and $V\left(\bar{\xi}_{\mathbb{Z}}\right)$, such that

$$
\Gamma\left(\xi_{\mathbb{Z}}, \lambda_{\mathbb{Z}}\right)=0_{\mathbb{Z}}
$$

has for all $\lambda_{\mathbb{Z}} \in U\left(\bar{\lambda}_{\mathbb{Z}}\right)$ a unique solution $\xi_{\mathbb{Z}} \in V\left(\bar{\xi}_{\mathbb{Z}}\right)$.

Proof: Since $\Gamma\left(\bar{\xi}_{\mathbb{Z}}, \bar{\lambda}_{\mathbb{Z}}\right)=0_{\mathbb{Z}}$, the assertion follows from the implicit function theorem, cf. Dieudonné (1969), if $D_{x} \Gamma\left(\bar{\xi}_{\mathbb{Z}}, \bar{\lambda}_{\mathbb{Z}}\right)$ is a homeomorphism of $X_{\mathbb{Z}}$ into itself.

Note that $u_{\mathbb{Z}}$ is a solution of $D_{x} \Gamma\left(\bar{\xi}_{\mathbb{Z}}, \bar{\lambda}_{\mathbb{Z}}\right) u_{\mathbb{Z}}=0_{\mathbb{Z}}$ if and only if

$$
u_{n+1}=D_{x} f\left(\bar{\xi}_{n}, \bar{\lambda}_{n}\right) u_{n}, \quad \text { for all } n \in \mathbb{Z} \text {. }
$$

From A1, A2 it follows that the matrices $D_{x} f\left(\bar{\xi}_{n}, \bar{\lambda}_{n}\right)$ and $D_{x} f\left(\bar{\xi}_{n}, \bar{\lambda}_{n}\right)^{-1}$ are uniformly bounded for $n \in \mathbb{Z}$. The difference equation (8) possesses by assumption A3 an exponential dichotomy on $\mathbb{Z}$ and as a consequence, $\mathcal{N}\left(D_{x} \Gamma\left(\bar{\xi}_{\mathbb{Z}}, \bar{\lambda}_{\mathbb{Z}}\right)\right)=\left\{0_{\mathbb{Z}}\right\}$, see (Palmer 1988, Section 2).

On the other hand, the inhomogeneous difference equation

$$
u_{n+1}=D_{x} f\left(\bar{\xi}_{n}, \bar{\lambda}_{n}\right) u_{n}+h_{n}, \quad n \in \mathbb{Z}
$$

possesses by (Palmer 1988, Lemma 2.7) for each sequence $h_{\mathbb{Z}} \in X_{\mathbb{Z}}$ a unique bounded solution on $\mathbb{Z}$. Thus $D_{x} \Gamma\left(\bar{\xi}_{\mathbb{Z}}, \bar{\lambda}_{\mathbb{Z}}\right)$ is also surjective and therefore it is a homeomorphism.

Let $\lambda_{\mathbb{Z}} \in U \subset U\left(\bar{\lambda}_{\mathbb{Z}}\right)$ and denote by $\xi_{\mathbb{Z}}$ the unique bounded solution of (4) in $V\left(\bar{\xi}_{\mathbb{Z}}\right)$. The next lemma shows that the variational equation

$$
u_{n+1}=D_{x} f\left(\xi_{n}, \lambda_{n}\right) u_{n}, \quad n \in \mathbb{Z}
$$

possesses an exponential dichotomy on $\mathbb{Z}$, if $U$ is chosen sufficiently small.

Lemma 3 Assume $\boldsymbol{A} \mathbf{1}-\boldsymbol{A}$ 3. Then neighborhoods $U$ of $\bar{\lambda}_{\mathbb{Z}}$ and $V$ of $\bar{\xi}_{\mathbb{Z}}$ exist, such that the difference equation

$$
u_{n+1}=D_{x} f\left(\varrho_{n}, \lambda_{n}\right) u_{n}, \quad n \in \mathbb{Z}
$$


possesses for $\varrho_{\mathbb{Z}} \in V, \lambda_{\mathbb{Z}} \in U$ an exponential dichotomy on $\mathbb{Z}$. The dichotomy constants do not depend on the specific sequences $\lambda_{\mathbb{Z}}, \varrho_{\mathbb{Z}}$.

The equation $\Gamma\left(\cdot, \lambda_{\mathbb{Z}}\right)=0$ possesses for each $\lambda_{\mathbb{Z}} \in U$ a unique solution $\xi_{\mathbb{Z}} \in V$ and (9) has an exponential dichotomy on $\mathbb{Z}$.

Proof: Due to assumption A3, the difference equation (8) has an exponential dichotomy on $\mathbb{Z}$. An application of the Roughness-Theorem 14 guarantees the existence of an exponential dichotomy of the perturbed equation

$$
u_{n+1}=\left(D_{x} f\left(\bar{\xi}_{n}, \bar{\lambda}_{n}\right)+\left[D_{x} f\left(\varrho_{n}, \lambda_{n}\right)-D_{x} f\left(\bar{\xi}_{n}, \bar{\lambda}_{n}\right)\right]\right) u_{n}, \quad n \in \mathbb{Z}
$$

if

$$
\left\|D_{x} f\left(\varrho_{n}, \lambda_{n}\right)-D_{x} f\left(\bar{\xi}_{n}, \bar{\lambda}_{n}\right)\right\| \leq \beta
$$

holds, where $\beta$ is specified in Theorem 14. The inequality (10) is satisfied if $V \subset$ $V\left(\bar{\xi}_{\mathbb{Z}}\right)$ and $U_{1} \subset U\left(\bar{\lambda}_{\mathbb{Z}}\right)$ are chosen sufficiently small.

Choose $U \subset U_{1} \subset U\left(\bar{\lambda}_{\mathbb{Z}}\right)$ such that for all $\lambda_{\mathbb{Z}} \in U$, the unique solution $\xi_{\mathbb{Z}}\left(\lambda_{\mathbb{Z}}\right)$ (Lemma 2) lies in $V$. From the first part of the proof, we immediately see that (9) possesses an exponential dichotomy.

The numerical computation of bounded trajectories is based on solving sparse boundary value problems. In Section 2.1 a general ansatz is introduced and approximation results are provided. Note that in non-autonomous systems, a boundary operator, restricting the end points, for example, to linearizations of stable and unstable fiber bundles must depend on time. Nevertheless we reduce the computational effort by choosing time-independent boundary conditions. This simplification is justified in Section 2.2 by showing that the difference between two solutions of (5) for different sequences $\lambda_{\mathbb{Z}}, \mu_{\mathbb{Z}}$ that coincide on some interval $J$, decreases exponentially fast towards the middle. For asymptotically constant $\mu_{\mathbb{Z}}$, an approximation theorem is stated in Section 2.3 and a combination of these results enables the computation of asymptotically non-constant trajectories with high accuracy, see Theorem 8 .

\subsection{A boundary value approach}

Let $\bar{\mu}_{\mathbb{Z}} \in U$; denote by $\bar{\zeta}_{\mathbb{Z}}$ the unique solution of

$$
\zeta_{n+1}=f\left(\zeta_{n}, \bar{\mu}_{n}\right), \quad n \in \mathbb{Z}
$$

in $V$ and by $P_{n}^{s}, P_{n}^{u}$ the dichotomy projectors of

$$
u_{n+1}=D_{x} f\left(\bar{\zeta}_{n}, \bar{\mu}_{n}\right) u_{n}, \quad n \in \mathbb{Z},
$$

see Lemma 3. For computing an approximation of $\bar{\zeta}_{\mathbb{Z}}$, we solve the boundary value problem

$$
\Gamma_{J}\left(x_{J}, \bar{\mu}_{J}\right):=\left(\left(x_{n+1}-f\left(x_{n}, \bar{\mu}_{n}\right)\right)_{n \in \tilde{J}}, b_{n_{ \pm}}\left(x_{n_{-}}, x_{n_{+}}\right)\right)=0_{J}
$$


where $J=\left[n_{-}, n_{+}\right]$and $\tilde{J}=\left[n_{-}, n_{+}-1\right]$ are finite intervals.

It is assumed that the boundary operator $b_{n_{ \pm}}$, which may depend on $n_{ \pm}$, fulfills the following conditions.

A4 $b_{n_{ \pm}} \in \mathcal{C}^{1}\left(\mathbb{R}^{2 k}, \mathbb{R}^{k}\right), D b_{n_{ \pm}}\left(x_{n_{-}}, x_{n_{+}}\right)$is uniformly bounded for $x_{\mathbb{Z}} \in U$ and uniformly Lipschitz w.r.t. $-n_{-}, n_{+} \in \mathbb{N}$.

A5 $b_{n_{ \pm}}\left(\bar{\zeta}_{n_{-}}, \bar{\zeta}_{n_{+}}\right) \rightarrow 0$ as $n_{ \pm} \rightarrow \pm \infty$.

A6 The matrix

$$
\left(D_{1} b_{n_{ \pm}}\left(\bar{\zeta}_{n_{-}}, \bar{\zeta}_{n_{+}}\right)\left|\mathcal{R}\left(P_{n_{-}}^{s}\right) \quad D_{2} b_{n_{ \pm}}\left(\bar{\zeta}_{n_{-}}, \bar{\zeta}_{n_{+}}\right)\right| \mathcal{R}\left(P_{n_{+}}^{u}\right)\right)
$$

possesses for sufficiently large $-n_{-}, n_{+}$a uniformly bounded inverse.

Note that the definition of a boundary operator, fulfilling A4-A6 requires knowledge about the whole infinite difference equation, i.e. the whole parameter sequence $\bar{\mu}_{\mathbb{Z}}$. In practical examples, one therefore defines $n_{ \pm}$-independent boundary conditions. Errors that occurs in this case from $\bar{\mu}_{n},|n|$ large are discussed in the forthcoming section. First, we consider the general $n$-dependent case and prove an approximation theorem.

Theorem 4 Assume $\boldsymbol{A 1 - \boldsymbol { A } 6}$. Then constants $\delta, N$ exist, such that the approximating system $\Gamma_{J}\left(x_{J}, \bar{\mu}_{J}\right)=0_{J}$, cf. (12) possesses a unique solution

$$
x_{J} \in B_{\delta}\left(\bar{\zeta}_{J}\right) \quad \text { for } J=\left[n_{-}, n_{+}\right],-n_{-}, n_{+} \geq N .
$$

With a J-independent constant $C>0$, the error can be estimated as

$$
\left\|\bar{\zeta}_{J}-x_{J}\right\| \leq C\left\|b_{n_{ \pm}}\left(\bar{\zeta}_{n_{-}}, \bar{\zeta}_{n_{+}}\right)\right\| .
$$

Proof: First, we show that $D_{1} \Gamma_{J}\left(\bar{\zeta}_{J}, \bar{\mu}_{J}\right)$ has for sufficiently large intervals $J$ a uniformly bounded inverse.

Let $\left(y_{\tilde{J}}, r\right) \in X_{\tilde{J}} \times \mathbb{R}^{k}$. Then the inhomogeneous equation $D_{1} \Gamma_{J}\left(\bar{\zeta}_{J}, \bar{\mu}_{J}\right) u_{J}=$ $\left(y_{\tilde{J}}, r\right)$ may equivalently be written as

$$
\begin{aligned}
u_{n+1}-D_{x} f\left(\bar{\zeta}_{n}, \bar{\mu}_{n}\right) u_{n} & =y_{n}, \quad n \in \tilde{J} \\
D_{1} b_{n_{ \pm}}\left(\bar{\zeta}_{n_{-}}, \bar{\zeta}_{n_{+}}\right) u_{n_{-}}+D_{2} b_{n_{ \pm}}\left(\bar{\zeta}_{n_{-}}, \bar{\zeta}_{n_{+}}\right) u_{n_{+}} & =r .
\end{aligned}
$$

Denote by $\Phi$ the solution operator of the homogeneous equation (11). Since this difference equation possesses by Lemma 3 an exponential dichotomy on $\mathbb{Z}$ with data $\left(K, \alpha, P_{n}^{s}, P_{n}^{u}\right)$, any solution of (14) has the form

$$
u_{n}=\Phi(n, 0) v+\sum_{m \in \tilde{J}} G(n, m+1) y_{m}, \quad n \in J,
$$


with some $v \in \mathbb{R}^{k}$. Here, $G$ denotes Green's function, defined as

$$
G(n, m)= \begin{cases}\Phi(n, m) P_{m}^{s}, & n \geq m \\ -\Phi(n, m) P_{m}^{u}, & n<m\end{cases}
$$

for which the estimates hold

$$
\begin{aligned}
\|G(n, m)\|=\left\|\Phi(n, m) P_{m}^{s}\right\| \leq K \mathrm{e}^{-\alpha(n-m)}, & \text { for } n \geq m, \\
\|G(n, m)\|=\left\|\Phi(n, m) P_{m}^{u}\right\| \leq K \mathrm{e}^{-\alpha(m-n)}, & \text { for } n<m .
\end{aligned}
$$

We introduce the following decomposition of $v$ :

$$
v=\Phi\left(0, n_{-}\right) v_{-}+\Phi\left(0, n_{+}\right) v_{+}, \quad v_{-} \in \mathcal{R}\left(P_{n_{-}}^{s}\right), v_{+} \in \mathcal{R}\left(P_{n_{+}}^{u}\right)
$$

In this notation, (15) reads

$$
\begin{aligned}
& D_{1} b_{n_{ \pm}}\left(\bar{\zeta}_{n_{-}}, \bar{\zeta}_{n_{+}}\right) v_{-}+D_{1} b_{n_{ \pm}}\left(\bar{\zeta}_{n_{-}}, \bar{\zeta}_{n_{+}}\right) \Phi\left(n_{-}, n_{+}\right) v_{+} \\
+ & D_{2} b_{n_{ \pm}}\left(\bar{\zeta}_{n_{-}}, \bar{\zeta}_{n_{+}}\right) \Phi\left(n_{+}, n_{-}\right) v_{-}+D_{2} b_{n_{ \pm}}\left(\bar{\zeta}_{n_{-}}, \bar{\zeta}_{n_{+}}\right) v_{+}=R,
\end{aligned}
$$

where

$$
\begin{aligned}
R= & r-D_{1} b_{n_{ \pm}}\left(\bar{\zeta}_{n_{-}}, \bar{\zeta}_{n_{+}}\right) \sum_{m \in \tilde{J}} G\left(n_{-}, m+1\right) y_{m} \\
& -D_{2} b_{n_{ \pm}}\left(\bar{\zeta}_{n_{-}}, \bar{\zeta}_{n_{+}}\right) \sum_{m \in \tilde{J}} G\left(n_{+}, m+1\right) y_{m} .
\end{aligned}
$$

Employing the dichotomy estimates and assumption A4, the second and third term in (19) converge exponentially fast to 0 as $n_{ \pm} \rightarrow \pm \infty$ and it follows from A6 that (19) has a unique solution for sufficiently large $-n_{-}, n_{+}$. From (17) and (18), we obtain with some $J$-independent generic constant $C>0$ the uniform estimate $\|R\| \leq C\left(\left\|y_{\tilde{J}}\right\|+\|r\|\right)$ and therefore $\left\|v_{ \pm}\right\| \leq C\left(\left\|y_{\tilde{J}}\right\|+\|r\|\right)$. Furthermore, using the dichotomy estimates it holds for $n_{-} \leq n \leq n_{+}$

$$
\begin{aligned}
\|\Phi(n, 0) v\| & \leq\left\|\Phi\left(n, n_{-}\right) P_{n_{-}}^{s}\right\|\left\|v_{-}\right\|+\left\|\Phi\left(n, n_{+}\right) P_{n_{+}}^{u}\right\|\left\|v_{+}\right\| \\
& \leq C\left(\left\|v_{-}\right\|+\left\|v_{+}\right\|\right) .
\end{aligned}
$$

Thus, a $J$-independent constant $\sigma$ exists, such that

$$
\left\|u_{J}\right\| \leq\|\Phi(n, 0) v\|+\sum_{m \in \tilde{J}}\|G(n, m+1)\|\left\|y_{m}\right\| \leq \sigma^{-1}\left(\left\|y_{\tilde{J}}\right\|+\|r\|\right),
$$

and consequently $\left\|D_{1} \Gamma_{J}\left(\bar{\zeta}_{J}, \bar{\mu}_{J}\right)^{-1}\right\| \leq \sigma^{-1}$.

The remaining part of the proof is an application of Lemma 15 with the setting

$$
Y=\left(X_{J},\|\cdot\|\right), \quad Z=\left(X_{\tilde{J}} \times \mathbb{R}^{k},\|\cdot\|+\|\cdot\|\right), \quad F=\Gamma_{J}\left(\cdot, \bar{\mu}_{J}\right), \quad y_{0}=\bar{\zeta}_{J} .
$$


We show that assumption (45) of Lemma 15 is fulfilled. By assumptions A1 and A4, there exists a $\delta>0$ such that

$$
\begin{aligned}
\left\|D_{1} \Gamma_{J}\left(z_{J}, \bar{\mu}_{J}\right)-D_{1} \Gamma_{J}\left(\bar{\zeta}_{J}, \bar{\mu}_{J}\right)\right\| \leq & \sup _{n \in \tilde{J}}\left\|D_{x} f\left(z_{n}, \bar{\mu}_{n}\right)-D_{x} f\left(\bar{\zeta}_{n}, \bar{\mu}_{n}\right)\right\| \\
& +\left\|D b_{n_{ \pm}}\left(z_{n_{-}}, z_{n_{+}}\right)-D b_{n_{ \pm}}\left(\bar{\zeta}_{n_{-}}, \bar{\zeta}_{n_{+}}\right)\right\| \leq \frac{\sigma}{2}
\end{aligned}
$$

for $z_{J} \in B_{\delta}\left(\bar{\zeta}_{J}\right)$.

Assumption (46) can also be verified with $\kappa=\frac{\sigma}{2}$ :

$$
\left\|\Gamma_{J}\left(\bar{\zeta}_{J}, \bar{\mu}_{J}\right)\right\|=\left\|\left(\bar{\zeta}_{n+1}-f\left(\bar{\zeta}_{n}, \bar{\mu}_{n}\right)\right)_{n \in \tilde{J}}\right\|+\left\|b_{n_{ \pm}}\left(\bar{\zeta}_{n_{-}}, \bar{\zeta}_{n_{+}}\right)\right\| \leq \frac{\sigma}{2} \delta
$$

holds due to assumption $\mathbf{A} 5$ for sufficiently large $-n_{-}, n_{+}$.

By Lemma 15 a unique solution $z_{J}$ of $\Gamma_{J}\left(z_{J}, \bar{\mu}_{J}\right)=0_{J}$ exists in $B_{\delta}\left(\bar{\zeta}_{J}\right)$ for $J$ sufficiently large, and an estimate of the approximation error follows from (48):

$$
\left\|\bar{\zeta}_{J}-z_{J}\right\| \leq \frac{1}{\sigma-\kappa}\left\|\Gamma_{J}\left(\bar{\zeta}_{J}, \bar{\mu}_{J}\right)-\Gamma_{J}\left(z_{J}, \bar{\mu}_{J}\right)\right\|=\frac{2}{\sigma}\left\|b_{n_{ \pm}}\left(\bar{\zeta}_{n_{-}}-\bar{\zeta}_{n_{+}}\right)\right\| .
$$

\subsection{Influence of $\bar{\lambda}_{n}$ with large $|n|$}

Assume that the sequence $\bar{\lambda}_{\mathbb{Z}}$ is given, cf. assumption A2. For computing a finite approximation $z_{J}$ of the bounded trajectory $\bar{\xi}_{\mathbb{Z}}$, we solve the boundary value problem (12) numerically. Doing so, the choice of a suitable boundary operator is crucial. Following the ideas, introduced in Hüls (2008), we obtain accurate approximations of dichotomy projectors $P_{n_{-}}^{s}$ and $P_{n_{+}}^{u}$ that can be used for defining projection boundary conditions. This ansatz requires solving large sparse linear systems. In this paper, we avoid this extra computations by taking $n_{ \pm}$-independent boundary conditions.

Obviously, the finite middle part of the sequence $\bar{\xi}_{\mathbb{Z}}$, denoted by $\bar{\xi}_{J}$, depends on $\bar{\lambda}_{J}$ but also on the parameters $\bar{\lambda}_{n}, n \notin J$. On the other hand, finite approximations, i.e. the solutions of the boundary value problem (12), coincide for all sequences $\bar{\mu}_{\mathbb{Z}}$ and $\bar{\lambda}_{\mathbb{Z}}$ fulfilling $\bar{\mu}_{n}=\bar{\lambda}_{n}$ for $n \in J$. Thus, in case of $n_{ \pm}$-independent boundary conditions, we will have a relatively large approximation error at the boundary. For numerical calculations it is convenient to choose periodic boundary conditions

$$
b_{\text {per }}(x, y):=x-y \text {. }
$$

First, we prove that the influence of the outer points decreases exponentially fast towards the middle of the interval $J$.

Theorem 5 Assume $\boldsymbol{A} \mathbf{1}-\boldsymbol{A}$ 3. Let $U$ and $V$ be given as in Lemma 3. Then there are positive constants $C$ and $\alpha$ such that for any interval $J$ and any $\lambda_{\mathbb{Z}}, \mu_{\mathbb{Z}} \in U$ with $\lambda_{n}=\mu_{n}$ for $n \in J$, the estimate

$$
\left\|\xi_{n}-\zeta_{n}\right\| \leq C\left(\mathrm{e}^{-\alpha\left(n-n_{-}\right)}+\mathrm{e}^{-\alpha\left(n_{+}-n\right)}\right), \quad n \in J
$$


holds. Here $\xi_{\mathbb{Z}}, \zeta_{\mathbb{Z}} \in V$ denote the bounded solutions w.r.t. $\lambda_{\mathbb{Z}}$ and $\mu_{\mathbb{Z}}$, respectively. Proof: W.l.o.g. assume that the components $V_{i}$ of $V$ are convex for all $i \in \mathbb{Z}$. Due to our assumptions it holds that

$$
\xi_{n+1}=f\left(\xi_{n}, \lambda_{n}\right) \quad \text { and } \quad \zeta_{n+1}=f\left(\zeta_{n}, \mu_{n}\right), \quad n \in \mathbb{Z} .
$$

Let $d_{\mathbb{Z}}=\zeta_{\mathbb{Z}}-\xi_{\mathbb{Z}}$ and $h_{\mathbb{Z}}=\mu_{\mathbb{Z}}-\lambda_{\mathbb{Z}}$. Then $d_{\mathbb{Z}}$ is a solution of the following difference equation

$$
\begin{aligned}
d_{n+1}= & f\left(\xi_{n}+d_{n}, \lambda_{n}+h_{n}\right)-f\left(\xi_{n}, \lambda_{n}\right) \\
= & f\left(\xi_{n}+d_{n}, \lambda_{n}\right)+\int_{0}^{1} D_{\lambda} f\left(\xi_{n}+d_{n}, \lambda_{n}+\tau h_{n}\right) \mathrm{d} \tau h_{n}-f\left(\xi_{n}, \lambda_{n}\right) \\
= & f\left(\xi_{n}, \lambda_{n}\right)+\int_{0}^{1} D_{x} f\left(\xi_{n}+\tau d_{n}, \lambda_{n}\right) \mathrm{d} \tau d_{n} \\
& +\int_{0}^{1} D_{\lambda} f\left(\xi_{n}+d_{n}, \lambda_{n}+\tau h_{n}\right) \mathrm{d} \tau h_{n}-f\left(\xi_{n}, \lambda_{n}\right) \\
= & \int_{0}^{1} D_{x} f\left(\xi_{n}+\tau d_{n}, \lambda_{n}\right) \mathrm{d} \tau d_{n}+\int_{0}^{1} D_{\lambda} f\left(\xi_{n}+d_{n}, \lambda_{n}+\tau h_{n}\right) \mathrm{d} \tau h_{n} .
\end{aligned}
$$

The homogeneous difference equation

$$
u_{n+1}=D_{x} f\left(\bar{\xi}_{n}, \bar{\lambda}_{n}\right) u_{n}, \quad n \in \mathbb{Z}
$$

possesses by assumption $\mathbf{A} \mathbf{3}$ an exponential dichotomy on $\mathbb{Z}$. Let $\beta>0$ be chosen as in the Roughness-Theorem 14. From the construction of $U$ and $V$ and (10), it follows that

$$
\sup _{n \in \mathbb{Z}}\left\|\int_{0}^{1} D_{x} f\left(\xi_{n}+\tau d_{n}, \lambda_{n}\right)-D_{x} f\left(\bar{\xi}_{n}, \bar{\lambda}_{n}\right) \mathrm{d} \tau\right\| \leq \sup _{n \in \mathbb{Z}} \int_{0}^{1} \beta \mathrm{d} \tau=\beta .
$$

Applying the Roughness-Theorem 14, we get an exponential dichotomy on $\mathbb{Z}$ of the difference equation

$$
u_{n+1}=A_{n} u_{n}, \quad A_{n}=\int_{0}^{1} D_{x} f\left(\xi_{n}+\tau d_{n}, \lambda_{n}\right) \mathrm{d} \tau, \quad n \in \mathbb{Z} .
$$

Let $\left(K, \alpha, P_{n}^{s}, P_{n}^{u}\right)$ be the corresponding dichotomy data and denote the solution operator of (22) by $\Phi$. Consider the inhomogeneous difference equation

$$
u_{n+1}=A_{n} u_{n}+r_{n}, \quad r_{n}=\int_{0}^{1} D_{\lambda} f\left(\xi_{n}+d_{n}, \lambda_{n}+\tau h_{n}\right) \mathrm{d} \tau h_{n}, \quad n \in \mathbb{Z} .
$$

The unique bounded solution of (23) on $\mathbb{Z}$ is

$$
u_{n}=\sum_{m \in \mathbb{Z}} G(n, m+1) r_{m},
$$


cf. Palmer (1988), where $G$ is Green's function, introduced in (16).

Note that due to our assumptions, $\left\|r_{n}\right\|$ is bounded from above by some constant $R$ for all $n \in \mathbb{Z}$ and $r_{n}=0$ for $n \in J=\left[n_{-}, n_{+}\right]$, since $\lambda_{n}=\mu_{n}$ for $n \in J$.

For $n \in J$ we derive an estimate of $\left\|u_{n}\right\|$ :

$$
\begin{aligned}
\left\|u_{n}\right\| & \leq \sum_{m \in \mathbb{Z}}\left\|G(n, m+1) r_{m}\right\| \\
& =\sum_{m=-\infty}^{n_{-}-1}\left\|G(n, m+1) r_{m}\right\|+\sum_{m=n_{+}+1}^{\infty}\left\|G(n, m+1) r_{m}\right\| \\
& =\sum_{m=-\infty}^{n_{-}-1}\left\|\Phi(n, m+1) P_{m+1}^{s} r_{m}\right\|+\sum_{m=n_{+}+1}^{\infty}\left\|\Phi(n, m+1) P_{m+1}^{u} r_{m}\right\| \\
& \left.\leq \sum_{m=-\infty}^{n_{-}-1} R K \mathrm{e}^{-\alpha(n-m-1)}+\sum_{m=n_{+}+1}^{\infty} R K \mathrm{e}^{-\alpha(m+1-n)}\right) \\
& =R K\left(\sum_{m=-\infty}^{0} \mathrm{e}^{-\alpha\left(n-m-n_{-}\right)}+\sum_{m=0}^{\infty} \mathrm{e}^{-\alpha\left(m+n_{+}+2-n\right)}\right) \\
& =\frac{R K}{1-\mathrm{e}^{-\alpha}}\left(\mathrm{e}^{-\alpha\left(n-n_{-}\right)}+\mathrm{e}^{-\alpha\left(n_{+}-n+2\right)}\right) .
\end{aligned}
$$

By construction, $d_{\mathbb{Z}}$ is the bounded solution of (23), thus the estimate

$$
\left\|d_{n}\right\| \leq C\left(\mathrm{e}^{-\alpha\left(n-n_{-}\right)}+\mathrm{e}^{-\alpha\left(n_{+}-n\right)}\right)
$$

holds for all $n \in J$ with constant $C=\frac{R K}{1-\mathrm{e}^{-\alpha}}$.

For two sequences of parameters that converge towards each other, the next proposition shows that the corresponding solutions approach each other.

Proposition 6 Assume $\boldsymbol{A} \mathbf{1}-\boldsymbol{A}$ 3. Let $U$ and $V$ be given as in Lemma 3. Let $\lambda_{\mathbb{Z}}$, $\mu_{\mathbb{Z}} \in U$ such that $\lim _{n \rightarrow \pm \infty}\left|\lambda_{n}-\mu_{n}\right|=0$. Denote by $\xi_{\mathbb{Z}}, \zeta_{\mathbb{Z}} \in V$ the corresponding solutions. Then

$$
\lim _{n \rightarrow \pm \infty}\left\|\xi_{n}-\zeta_{n}\right\|=0 .
$$

Proof: Let $d_{\mathbb{Z}}=\zeta_{\mathbb{Z}}-\xi_{\mathbb{Z}}$ and $h_{\mathbb{Z}}=\mu_{\mathbb{Z}}-\lambda_{\mathbb{Z}}$. The same computations as in the proof of Theorem 5 yield for each $n \in \mathbb{Z}$

$$
d_{n}=\sum_{m \in \mathbb{Z}} G(n, m+1) r_{m}
$$

where $r_{\mathbb{Z}}$ is defined in (23) and $G$ is Green's function from (16) w.r.t. the difference equation (22). 
Exploiting (17), (18), the following estimates hold for $n \in \mathbb{Z}$ :

$$
\begin{aligned}
\left\|d_{n}\right\| & \leq \sum_{m \in \mathbb{Z}}\left\|G(n, m+1) r_{m}\right\| \\
& =\sum_{m=-\infty}^{n-1}\left\|\Phi(n, m+1) P_{m+1}^{s} r_{m}\right\|+\sum_{m=n}^{\infty}\left\|\Phi(n, m+1) P_{m+1}^{u} r_{m}\right\| \\
& \leq \sum_{m=-\infty}^{n-1} K \mathrm{e}^{-\alpha(n-m-1)}\left\|r_{m}\right\|+\sum_{m=n}^{\infty} K \mathrm{e}^{-\alpha(m+1-n)}\left\|r_{m}\right\| \\
& =\sum_{m=-\infty}^{0} K \mathrm{e}^{\alpha m}\left\|r_{m+n-1}\right\|+\sum_{m=1}^{\infty} K \mathrm{e}^{-\alpha m}\left\|r_{m+n-1}\right\| .
\end{aligned}
$$

Let $\varepsilon>0$. Since $r_{\mathbb{Z}}$ is bounded from above, a constant $M \in \mathbb{N}$ that does not depend on $n$ exists, such that

$$
\sum_{m=-\infty}^{-M-1} K \mathrm{e}^{\alpha m}\left\|r_{m+n-1}\right\|+\sum_{m=M+1}^{\infty} K \mathrm{e}^{-\alpha m}\left\|r_{m+n-1}\right\| \leq \frac{\varepsilon}{2}
$$

We show that also the middle part can be estimated by $\frac{\varepsilon}{2}$. Let

$$
C:=\sum_{m=-M}^{0} K \mathrm{e}^{\alpha m}+\sum_{m=1}^{M} K \mathrm{e}^{-\alpha m}
$$

and recall that $\left\|r_{n}\right\| \rightarrow 0$ as $n \rightarrow \pm \infty$. Thus a constant $N \in \mathbb{N}$ exists such that

$$
\left\|r_{m+n-1}\right\| \leq \frac{\varepsilon}{2 C} \quad \text { for all }|n| \geq N, m \in[-M, M]
$$

and as a consequence $\left\|d_{n}\right\| \leq \frac{\varepsilon}{2}+C \frac{\varepsilon}{2 C}=\varepsilon$ for all $|n| \geq N$.

\subsection{Approximation of bounded trajectories with asymptot- ically constant tails}

From the previous section, we know that for two given sequences $\bar{\lambda}_{\mathbb{Z}}, \bar{\mu}_{\mathbb{Z}}$ that coincide on the interval $J$, the corresponding solutions $\bar{\xi}_{\mathbb{Z}}, \bar{\zeta}_{\mathbb{Z}}$ of (5) are exponentially close in the middle of $J$. On the other hand, the solution of the boundary value problem (12) does not depend on $\bar{\lambda}_{n}$ for $n \notin J$.

In this section, we consider the case of an asymptotically autonomous system and show that Theorem 4 applies and allows the computation of accurate finite orbit segments. We assume that the asymptotically constant case is included in our neighborhoods. 
A7 There exists a sequence $\bar{\mu}_{\mathbb{Z}} \in U$ with corresponding solution $\bar{\zeta}_{\mathbb{Z}} \in V$ of (5), such that

$$
\lim _{n \rightarrow+\infty} \bar{\mu}_{n}=\lim _{n \rightarrow-\infty} \bar{\mu}_{n}=: \bar{\mu} \text { and } \lim _{n \rightarrow+\infty} \bar{\zeta}_{n}=\lim _{n \rightarrow-\infty} \bar{\zeta}_{n}=: \bar{\zeta}
$$

hold for some $\bar{\mu} \in \mathbb{R}, \bar{\zeta} \in \mathbb{R}^{k}$.

Note that if $\bar{\lambda}_{\mathbb{Z}}$ and $\bar{\xi}_{\mathbb{Z}}$, introduced in assumption A2, do not vary too much as $n \rightarrow \pm \infty$, sequences $\bar{\mu}_{\mathbb{Z}} \in U, \bar{\zeta}_{\mathbb{Z}} \in V$ exist that satisfy (25). In several applications, condition $\mathbf{A} 7$ is naturally fulfilled. Let $\bar{\zeta}$ be a hyperbolic fixed point of $f(\cdot, \bar{\mu})$. For the constant sequence $\bar{\mu}_{\mathbb{Z}}$ defined as $\bar{\mu}_{n}=\bar{\mu}$, the constant trajectory $\bar{\zeta}_{\mathbb{Z}}\left(\bar{\zeta}_{n}=\right.$ $\bar{\zeta})$ obviously is a solution of (5). By Lemma 2 a generally non-constant bounded trajectory $\bar{\xi}_{\mathbb{Z}}$ exists for $\bar{\lambda}_{\mathbb{Z}} \in U\left(\bar{\mu}_{\mathbb{Z}}\right)$, fulfilling $\mathbf{A} \mathbf{3}$, see the first example in Section 4.

We assume that $\bar{\mu}_{\mathbb{Z}}$ is given as in assumption $\mathbf{A} 7$ and compute a finite segment of the bounded trajectory $\bar{\zeta}_{\mathbb{Z}}$.

Corollary 7 Assume $\boldsymbol{A 1 - A 3 , A 7 . ~ T h e n ~ c o n s t a n t s ~} \delta, N$ exist, such that the approximating system $\Gamma_{J}\left(z_{J}, \bar{\mu}_{J}\right)=0_{J}$ with periodic boundary conditions (20), possesses a unique solution

$$
z_{J} \in B_{\delta}\left(\bar{\zeta}_{J}\right) \quad \text { for } J=\left[n_{-}, n_{+}\right],-n_{-}, n_{+} \geq N .
$$

With a J-independent constant $C>0$, the error can be estimated as

$$
\left\|\bar{\zeta}_{J}-z_{J}\right\| \leq C\left\|\bar{\zeta}_{n_{-}}-\bar{\zeta}_{n_{+}}\right\|
$$

Proof: In order to apply Theorem 4, we verify assumptions A4-A6. Obviously, A4 holds and by assumption A7 $b\left(\bar{\zeta}_{n_{-}}, \bar{\zeta}_{n_{+}}\right) \rightarrow 0$ as $\pm n_{ \pm} \rightarrow \infty$. Furthermore,

$$
\left(D_{1} b\left(\bar{\zeta}_{n_{-}}, \bar{\zeta}_{n_{+}}\right)_{\mid \mathcal{R}\left(P_{n_{-}}^{s}\right)} \quad D_{2} b\left(\bar{\zeta}_{n_{-}}, \bar{\zeta}_{n_{+}}\right)_{\mid \mathcal{R}\left(P_{n_{+}}^{u}\right)}\right)=\left(\begin{array}{ll}
I_{\mid \mathcal{R}\left(P_{n_{-}}^{s}\right)} & \left.-I_{\mid \mathcal{R}\left(P_{n_{+}}^{u}\right)}\right)
\end{array}\right.
$$

has a uniformly bounded inverse, since the angle between range and nullspace of the dichotomy projectors of the difference equation $u_{n+1}=D_{x} f\left(\bar{\zeta}_{n}, \bar{\mu}_{n}\right) u_{n}$ is uniformly bounded from below $\left(\left\|D_{x} f\left(\bar{\zeta}_{n_{-}}, \bar{\mu}_{n_{-}}\right)-D_{x} f\left(\bar{\zeta}_{n_{+}}, \bar{\mu}_{n_{+}}\right)\right\| \rightarrow 0\right.$ as $\left.\pm n_{ \pm} \rightarrow \infty\right)$. Therefore, Theorem 4 applies and guarantees existence of a unique bounded solution $z_{J}$, fulfilling

$$
\left\|\bar{\zeta}_{J}-z_{J}\right\| \leq C\left\|b\left(\bar{\zeta}_{n_{-}}, \bar{\zeta}_{n_{+}}\right)\right\|=C\left\|\bar{\zeta}_{n_{-}}-\bar{\zeta}_{n_{+}}\right\|
$$

\subsection{Approximation of bounded trajectories with varying tails}

Combining the previous results, we show that one obtains an approximation of the trajectory $\bar{\xi}_{\mathbb{Z}} \in V$ on the finite interval $J$ that is accurate up to any given accuracy $\Delta$. 
Theorem 8 Assume $\boldsymbol{A} \mathbf{1}-\boldsymbol{A} 3, \boldsymbol{A}$ 7. Let $J=\left[n_{-}, n_{+}\right]$be a finite interval and let $\Delta$ be a given accuracy. Denote by $\bar{\xi}_{\mathbb{Z}}$ the solution of (5) with respect to the parameters $\bar{\lambda}_{\mathbb{Z}}$, see $\boldsymbol{A} \mathcal{2}$, and let $\bar{\mu}_{\mathbb{Z}}$ be the sequence of parameters from $\boldsymbol{A} \boldsymbol{7}$. Consider the parameters

$$
\bar{\omega}_{n}(I)=\left\{\begin{array}{ll}
\bar{\lambda}_{n}, & \text { for } n \in I, \\
\bar{\mu}_{n}, & \text { for } n \notin I,
\end{array}, \text { where } I \subset \mathbb{Z}\right. \text { is an interval, }
$$

with corresponding solution $\bar{\eta}_{\mathbb{Z}}(I)$ of

$$
z_{n+1}=f\left(z_{n}, \bar{\omega}_{n}(I)\right), \quad n \in \mathbb{Z} .
$$

Then there exist intervals $\hat{J} \supset \bar{J} \supset J$ and $a \delta>0$, such that the difference equation

$$
\Gamma_{\hat{J}}\left(z_{\hat{J}}, \bar{\omega}_{\hat{J}}(\bar{J})\right)=0_{J} \text { with periodic boundary conditions }
$$

possesses a unique solution $z_{\hat{J}} \in B_{\delta}\left(\bar{\eta}_{\hat{J}}(\bar{J})\right)$, satisfying

$$
\left\|\bar{\xi}_{n}-z_{n}\right\| \leq \Delta \text { for all } n \in J
$$

Proof: The main idea is to compute an approximation of the orbit on a longer interval. Since the largest approximation errors occur at the boundary of this interval, we only take the accurate middle part. Let $\mu_{\mathbb{Z}}$ and $\zeta_{\mathbb{Z}}$ be given as in $\mathbf{A 7}$. Applying Lemma 2, it follows that (27) possesses in $V$ a unique solution $\bar{\eta}_{\mathbb{Z}}(I)$. By construction

$$
\lim _{n \rightarrow \pm \infty}\left|\bar{\omega}_{n}(I)-\bar{\mu}_{n}\right|=0
$$

and due to Proposition 6, the corresponding orbit also converges:

$$
\lim _{n \rightarrow \pm \infty}\left\|\bar{\eta}_{n}(I)-\bar{\zeta}_{n}\right\|=0
$$

as a consequence, $\left(\bar{\omega}_{\mathbb{Z}}(I), \bar{\eta}_{\mathbb{Z}}(I)\right)$ satisfies $\mathbf{A} 7$ for all finite intervals $I$.

Applying Theorem 5, we find an interval $\bar{J} \supset J$ such that

$$
\left\|\bar{\xi}_{n}-\bar{\eta}_{n}(\bar{J})\right\| \leq \frac{\Delta}{2} \text { holds for all } n \in J .
$$

In the second step, we compute a finite approximation of $\bar{\eta}_{\mathbb{Z}}(\bar{J})$. By Corollary 7 there exists an interval $\hat{J}=\left[\hat{n}_{-}, \hat{n}_{+}\right]$, such that the boundary value problem $\Gamma_{\hat{J}}\left(z_{\hat{J}}, \bar{\omega}_{\hat{J}}(\bar{J})\right)=0_{J}$ with periodic boundary conditions, possesses a unique solution $z_{\hat{J}} \in B_{\delta}\left(\bar{\eta}_{\hat{J}}(\bar{J})\right)$, fulfilling

$$
\left\|\bar{\eta}_{\hat{J}}(\bar{J})-z_{\hat{J}}\right\| \leq C\left\|\bar{\eta}_{\hat{n}_{-}}(\bar{J})-\bar{\eta}_{\hat{n}_{+}}(\bar{J})\right\|
$$

where the constant $C$ does not depend on the chosen interval $\hat{J}$. Since $\bar{\eta}_{\mathbb{Z}}(\bar{J})$ converges in the sense of (25), we can enlarge $\hat{J}$ such that

$$
\left\|\bar{\eta}_{\hat{n}_{-}}(\bar{J})-\bar{\eta}_{\hat{n}_{+}}(\bar{J})\right\| \leq \frac{\Delta}{2 C}
$$


holds and consequently

$$
\left\|\bar{\eta}_{\hat{J}}(\bar{J})-z_{\hat{J}}\right\| \leq \frac{\Delta}{2}
$$

Combining the results (28) and (29), we get for $n \in J$

$$
\left\|\bar{\xi}_{n}-z_{n}\right\| \leq\left\|\bar{\xi}_{n}-\bar{\eta}_{n}(\bar{J})\right\|+\left\|\bar{\eta}_{n}(\bar{J})-z_{n}\right\| \leq \frac{\Delta}{2}+\frac{\Delta}{2}=\Delta .
$$

Thus, the middle part $z_{J}$ is a finite approximation of $\bar{\xi}_{\mathbb{Z}}$ on the interval $J$ with accuracy $\Delta$.

The concrete choice of these buffer intervals for numerical computations is discussed in Section 4.2.

\section{Homoclinic trajectories}

Applying the approach introduce in the previous section, we obtain for a given parameter sequence $\bar{\lambda}_{\mathbb{Z}}$ a finite approximation of the bounded trajectory $\bar{\xi}_{\mathbb{Z}}$ of (5). In the following, we derive an algorithm for computing a second trajectory $\bar{x}_{\mathbb{Z}}$ that is homoclinic to the first one, see Definition 1. First, we assume existence as well as transversality.

A8 Let $\bar{\lambda}_{\mathbb{Z}}$ be given as in A2. For this parameter sequence a solution $\bar{x}_{\mathbb{Z}}$ of

$$
x_{n+1}=f\left(x_{n}, \bar{\lambda}_{n}\right), \quad n \in \mathbb{Z}
$$

exists, that is homoclinic to $\bar{\xi}_{\mathbb{Z}}$ and non-trivial, i.e. $\bar{x}_{\mathbb{Z}} \neq \bar{\xi}_{\mathbb{Z}}$.

A9 The trajectory $\bar{x}_{\mathbb{Z}}$ is transversal, i.e.

$$
u_{n+1}=D_{x} f\left(\bar{x}_{n}, \bar{\lambda}_{n}\right) u_{n}, n \in \mathbb{Z} \text { for } u_{\mathbb{Z}} \in X_{\mathbb{Z}} \quad \Longleftrightarrow \quad u_{\mathbb{Z}}=0_{\mathbb{Z}}
$$

First, we prove that the exponential dichotomy of the variational equation

$$
u_{n+1}=D_{x} f\left(\bar{\xi}_{n}, \bar{\lambda}_{n}\right) u_{n}, \quad n \in \mathbb{Z}
$$

implies an exponential dichotomy on $\mathbb{Z}$ of the difference equation

$$
u_{n+1}=D_{x} f\left(\bar{x}_{n}, \bar{\lambda}_{n}\right) u_{n}, \quad n \in \mathbb{Z} .
$$

Lemma 9 Assume $\boldsymbol{A} \mathbf{1}-\boldsymbol{A} 3, \boldsymbol{A 8}, \boldsymbol{A 9}$. Then the difference equation (31) possesses an exponential dichotomy on $\mathbb{Z}$. 
Proof: Since (30) possesses an exponential dichotomy on $\mathbb{Z}$ and $\left\|\bar{x}_{n}-\bar{\xi}_{n}\right\| \rightarrow 0$ as $n \rightarrow \pm \infty$, there exists an $N>0$, such that

$$
\left\|D_{x} f\left(\bar{x}_{n}, \bar{\lambda}_{n}\right)-D_{x} f\left(\bar{\xi}_{n}, \bar{\lambda}_{n}\right)\right\|<\beta, \quad \text { for all }|n| \geq N,
$$

where $\beta$ is the bound for the additive perturbation in Theorem 14. Thus, (31) possesses exponential dichotomies on $(-\infty,-N]$ and on $[N, \infty)$ which can be extended to $\mathbb{Z}^{-}$and $\mathbb{Z}^{+}$, respectively.

Due to our transversality assumption A9, the two half-sided dichotomies lead to an exponential dichotomy on $\mathbb{Z}$, cf. Palmer (1988).

After these preparations, we introduce techniques for the numerical approximation of the second trajectory $\bar{x}_{\mathbb{Z}}$. The main idea is to transform the system (5) into a topologically equivalent form, see Aulbach \& Wanner (2003), where we assume the family of transformations to be equicontinuous.

Definition 10 Two non-autonomous discrete time dynamical systems

$$
\begin{array}{ll}
x_{n+1}=f_{n}\left(x_{n}\right), & n \in \mathbb{Z}, \\
y_{n+1}=g_{n}\left(y_{n}\right), & n \in \mathbb{Z}
\end{array}
$$

are topologically equivalent if a family of homeomorphism $\left(T_{n}\right)_{n \in \mathbb{Z}}$ exist, such that $T_{n}$ and $T_{n}^{-1}$ are equicontinuous on any compact set and such that

$$
g_{n}=T_{n+1}^{-1} \circ f_{n} \circ T_{n}
$$

Orbits of topologically equivalent systems transform into each other as follows. Let $x_{\mathbb{Z}}$ be an orbit of (32), then $y_{n}=T_{n}^{-1}\left(x_{n}\right), n \in \mathbb{Z}$ defines an orbit of (33).

Note that equicontinuity in Definition 10 guarantees that homoclinic trajectories of (32) transform into homoclinic trajectories of (33) via $T_{n}^{-1}, n \in \mathbb{Z}$ and vice versa.

The following lemma introduces a topologically equivalent system to (5), having zero as an $n$-independent fixed point.

Lemma 11 Assume A1, A2 and let

$$
g_{n}(y):=T_{n+1}^{-1} \circ f\left(\cdot, \bar{\lambda}_{n}\right) \circ T_{n}(y)
$$

where $T_{n}(y):=y+\bar{\xi}_{n}$ for $n \in \mathbb{Z}$.

The difference equations (5) and

$$
y_{n+1}=g_{n}\left(y_{n}\right), \quad n \in \mathbb{Z}
$$

are topologically equivalent and 0 is an $n$-independent fixed point of (34). 
As a consequence, the task of computing a second trajectory of (5) that is homoclinic to $\bar{\xi}_{\mathbb{Z}}$ is equivalent to the computation of a homoclinic orbit $\bar{y}_{\mathbb{Z}}$ of (34) w.r.t. the fixed point 0 . The transformed system (34) is often called equation of perturbed motion.

Note that the variational equations (31) and

$$
u_{n+1}=D g_{n}\left(\bar{y}_{n}\right) u_{n}, \quad n \in \mathbb{Z}
$$

coincide. Consequently, (35) possesses an exponential dichotomy with the same data as (31) and the transversality assumption A9 holds for the transformed system, too. For system (34) the geometric interpretation of $\mathbf{A 9}$ is a transversal intersection of the corresponding stable and unstable fiber bundles of the fixed point 0, cf. (Hüls 2006, Lemma 3.7).

We obtain a finite approximation of the homoclinic orbit $\bar{y}_{\mathbb{Z}}$ on the interval $J$ by solving

$$
\Gamma_{J}\left(y_{J}\right)=\left(\left(y_{n+1}-g_{n}\left(y_{n}\right)\right)_{n \in \tilde{J}}, b_{n_{ \pm}}\left(y_{n_{-}}, y_{n_{+}}\right)\right)=0_{J},
$$

with boundary operator $b_{n_{ \pm}} \in \mathcal{C}^{1}\left(\mathbb{R}^{2 k}, \mathbb{R}^{k}\right)$. Similar to Section 2.3 , we do not construct a boundary operator, restricting the end points to linearizations of the corresponding stable and unstable fiber bundles. These fiber bundles are generally not known explicitly, cf. Hirsch et al. (1977) for the case of normally hyperbolic manifolds. Furthermore, the effort of approximating linearizations of these objects numerically, using the techniques introduced in Hüls (2008), is not justified by a slightly better rate of convergence of the algorithm, described above.

We choose boundary conditions that do not depend on $n_{ \pm}$, namely periodic boundary conditions, introduced in (20) or projection boundary conditions w.r.t. a fixed projector, defined as

$$
b(x, y):=\left(\begin{array}{c}
Y_{s}^{T} x \\
Y_{u}^{T} y
\end{array}\right), \quad x, y \in \mathbb{R}^{k}
$$

where the columns of $Y_{s}$ and $Y_{u}$ form an orthogonal basis of $\mathcal{N}(P)^{\perp}$ and $\mathcal{R}(P)^{\perp}$ with respect to a given projector $P$.

Theorem 4, applies to (36) if the boundary operator satisfies $\mathbf{A 4} \mathbf{4} \mathbf{A 6}$, which is guaranteed by imposing the following angle conditions, see Hüls (2008).

A10 There exists a $0<\sigma<\frac{\pi}{2}$ such that

- in case of periodic boundary conditions

$$
\measuredangle\left(\mathcal{R}\left(P_{n_{-}}^{s}\right), \mathcal{R}\left(P_{n_{+}}^{u}\right)\right)>\sigma
$$

holds for sufficiently large $-n_{-}, n_{+}$.

- in case of projection boundary conditions let $\operatorname{rank}(P)=\operatorname{rank}\left(P_{n}^{s}\right)$ and

$$
\measuredangle\left(\mathcal{R}\left(P_{n_{-}}^{s}\right), \mathcal{N}(P)\right)>\sigma, \quad \measuredangle\left(\mathcal{R}\left(P_{n_{+}}^{u}\right), \mathcal{R}(P)\right)>\sigma
$$

hold for sufficiently large $-n_{-}, n_{+}$. 
The angle between two subspaces $A$ and $B$ is defined as, see Golub \& Van Loan (1996)

$$
\measuredangle(A, B)=\theta \in\left[0, \frac{\pi}{2}\right], \quad \text { where } \quad \cos \theta=\max _{u \in A,\|u\|=1} \max _{v \in B,\|v\|=1} u^{T} v .
$$

The following corollary to Theorem 4 summarizes (local) existence and wellposedness results.

Corollary 12 Assume $\boldsymbol{A} \mathbf{1}-\boldsymbol{A} 3, \boldsymbol{A} \boldsymbol{8}-\boldsymbol{A} \mathbf{1 0}$. There exist constants $\delta, N$, such that the approximating system (36) $\Gamma_{J}\left(y_{J}\right)=0_{J}$ possesses a unique solution

$$
y_{J} \in B_{\delta}\left(\bar{y}_{\mid J}\right) \quad \text { for all } J=\left[n_{-}, n_{+}\right],
$$

where $-n_{-}, n_{+} \geq N$. With a J-independent constant $C>0$, the approximation error can be estimated as

$$
\left\|\bar{y}_{\mid J}-y_{J}\right\| \leq C\left\|b\left(\bar{y}_{n_{-}}, \bar{y}_{n_{+}}\right)\right\| .
$$

Transforming $y_{J}$ back to the original coordinates, we obtain a finite approximation

$$
x_{n}:=T_{n}\left(y_{n}\right)=y_{n}+\bar{\xi}_{n}, \quad n \in J
$$

of $\bar{x}_{\mathbb{Z}}$, fulfilling the same error estimate (38).

\section{Examples}

For an illustration of our approach, we approximate homoclinic trajectories for two examples. First, we apply our techniques to the well known Hénon-map, where we randomize one parameter to get a non-autonomous system. The second example is a predator-prey model, cf. Beddington et al. (1975), Murray (2002). In this model, the carrying capacity of the environment fluctuates in time and generates a nonautonomous system.

\subsection{The Hénon-map}

Hénon's map, cf. Mira (1987), Devaney (1989), Hale \& Koçak (1991) is defined as

$$
x_{n+1}=\left(\begin{array}{l}
\left(x_{1}\right)_{n+1} \\
\left(x_{2}\right)_{n+1}
\end{array}\right)=h\left(\left(\begin{array}{l}
\left(x_{1}\right)_{n} \\
\left(x_{2}\right)_{n}
\end{array}\right), \lambda, b\right):=\left(\begin{array}{c}
1+\left(x_{2}\right)_{n}-\lambda\left(x_{1}\right)_{n}^{2} \\
b\left(x_{1}\right)_{n}
\end{array}\right), \quad n \in \mathbb{Z} .
$$

Fix the parameter $b=0.3$. This map possesses for $\lambda>-\frac{(b-1)^{2}}{4}$ the fixed point

$$
\eta(\lambda, b)=\left(\begin{array}{c}
z(\lambda, b) \\
b z(\lambda, b)
\end{array}\right), \text { where } z(\lambda, b)=\frac{b-1+\sqrt{(b-1)^{2}+4 \lambda}}{2 \lambda} .
$$


For fixed parameter $\hat{\lambda}=1.5$, the matrix

$$
A=D_{x} h(\eta(\hat{\lambda}, b), \hat{\lambda}, b)=\left(\begin{array}{cc}
-b+1-\sqrt{(b-1)^{2}+4 \hat{\lambda}} & 1 \\
b & 0
\end{array}\right)
$$

has the eigenvalues $\sigma_{s} \approx 0.15$ and $\sigma_{u} \approx-1.998$ and consequently $A$ is hyperbolic and the difference equation

$$
u_{n+1}=A u_{n}, \quad n \in \mathbb{Z}
$$

possesses an exponential dichotomy on $\mathbb{Z}$.

Furthermore, a transversal homoclinic orbit $x_{\mathbb{Z}}$ w.r.t. the fixed point $\eta(\hat{\lambda}, b)$ exists, cf. Beyn et al. (2004). Theorem 14 applies with the setting $A_{n}=A$ and $B_{n}=D_{x} h\left(x_{n}, \hat{\lambda}, b\right)-A$, and it follows that

$$
u_{n+1}=D_{x} h\left(x_{n}, \hat{\lambda}, b\right) u_{n}, \quad n \in \mathbb{Z}
$$

has exponential dichotomies on $\mathbb{Z}^{-}$and $\mathbb{Z}^{+}$which could, due to transversality, be combined to an exponential dichotomy on $\mathbb{Z}$.

In the language of this paper, $x_{\mathbb{Z}}$ and $\xi_{\mathbb{Z}}$, where $\xi_{n}=\eta(\hat{\lambda}, b)$ for all $n \in \mathbb{Z}$, are two homoclinic trajectories. From Lemma 2 and Lemma 3, the existence of a neighborhood $U\left(\hat{\lambda}_{\mathbb{Z}}\right)$ follows, in which the non-autonomous system

$$
x_{n+1}=h\left(x_{n}, \bar{\lambda}_{n}, b\right), \quad n \in \mathbb{Z}, \quad \bar{\lambda}_{\mathbb{Z}} \in U\left(\hat{\lambda}_{\mathbb{Z}}\right)
$$

possesses two bounded homoclinic trajectories $\bar{\xi}_{\mathbb{Z}}$ and $\bar{x}_{\mathbb{Z}}$, and the corresponding variational equations exhibit exponential dichotomies. As a consequence, our assumptions A1-A3, A7-A9 are satisfied for $\lambda_{\mathbb{Z}} \in U\left(\hat{\lambda}_{\mathbb{Z}}\right)$.

For the forthcoming numerical computations, we choose an interval around $\hat{\lambda}=$ 1.5 , namely $I=[1,2]$, and take a sequence $\lambda_{\mathbb{Z}} \in I^{\mathbb{Z}}$ at random.

\subsection{Computation of a bounded trajectory}

First, we approximate the bounded trajectory $\bar{\xi}_{\mathbb{Z}}$ on the finite interval $J=\left[n_{-}, n_{+}\right]$ by computing a longer orbit segment $\xi_{\bar{J}}$ on $\bar{J}=\left[\bar{n}_{-}, \bar{n}_{+}\right]$and taking only the accurate middle part $\xi_{J}$ as suggested in Section 2.4.

For illustrating that errors at the boundary decay exponentially fast toward the middle of $J$, we choose two sequences at random on the interval $[-40,40]$ that coincide in the middle interval $[-20,20]$. The solutions of the boundary value problem (12), (20) are shown in Figure 1. 

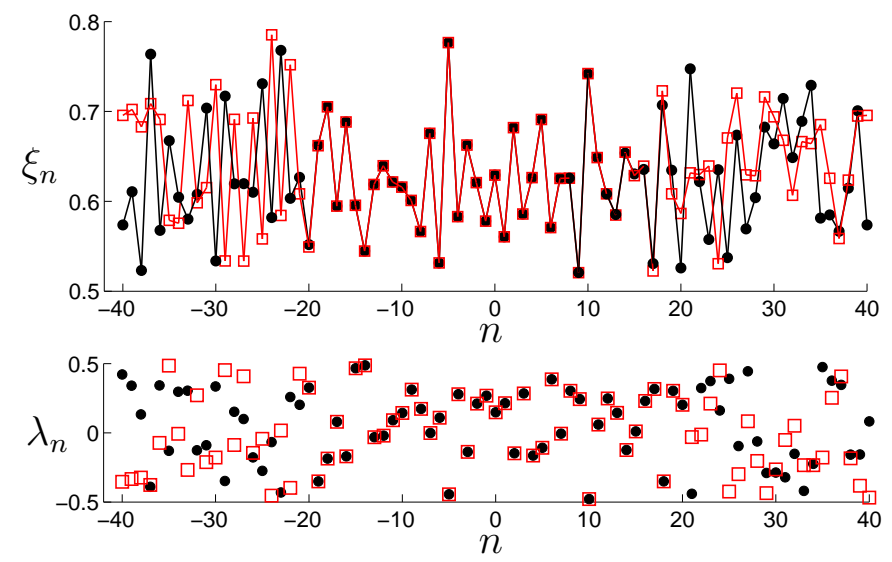

Figure 1: For two randomly chosen parameter sequences with the same middle part (lower diagram), the upper picture shows the corresponding solutions of (12), (20).

The choice of $\bar{n}_{ \pm}$is guided by Theorem 5 . We take $\bar{n}_{ \pm}$such that the difference between two solutions with different tails is of the order $\Delta$, i.e.

$$
\mathrm{e}^{-\alpha_{-}\left(n_{-}-\bar{n}_{-}\right)}+\mathrm{e}^{-\alpha_{+}\left(\bar{n}_{+}-n_{+}\right)}=\mathcal{O}(\Delta) .
$$

Here $\alpha_{ \pm}$denote the dichotomy constants w.r.t. the stable and unstable direction and $\Delta=10^{-16}$ is the machine accuracy. Let

$$
\bar{n}_{-}=\left\lfloor n_{-}+\frac{\log \Delta}{\alpha_{-}}\right\rfloor \quad \text { and } \quad \bar{n}_{+}=\left\lceil n_{+}-\frac{\log \Delta}{\alpha_{+}}\right\rceil .
$$

As a guess of $\alpha_{ \pm}$, we take into account the weakest rates in the stable and unstable directions and define $\alpha_{-}=\log \left|\sigma_{s}\right|$ and $\alpha_{+}=\log \left|\sigma_{u}\right|$, cf. (24).

For testing the validity of this ansatz, let $\bar{J}=[-100,100], \hat{J}=[-150,150]$ and choose a sequence $\lambda_{\hat{J}} \in I^{\hat{J}}$ at random. Then, a second sequence $\mu_{\hat{J}}$ is defined, such that $\mu_{\bar{J}}=\lambda_{\bar{J}}$ holds.

With respect to the parameter sequences $\lambda_{\hat{J}}, \mu_{\hat{J}}$, we compute the associated bounded trajectories $\xi_{\hat{J}}, \zeta_{\hat{J}}$, respectively, using Newton's method for solving the non-linear systems. As an initial guess $x_{\hat{J}}$, we take the fixed points $x_{n}=\eta\left(\lambda_{n}, b\right)$ for $n \in \hat{J}$. For an illustration, $d_{n}:=\left\|\xi_{n}-\zeta_{n}\right\|$ is plotted over $n$ in a logarithmic scale. In Figure 2, these computations are performed for 10 sequences $\mu_{\hat{J}}$, having different, randomly chosen tails. Define $J=\left[n_{-}, n_{+}\right]$, where $n_{ \pm}$are given in (41). We expect that the influence of parameter values outside the interval $\bar{J}$ on the solution in $J$, is of magnitude $\mathcal{O}(\Delta)$. For an illustration, two lines are drawn, connecting the points $\left(\bar{n}_{-}, \frac{1}{2}\right)$ with $\left(n_{-}, \Delta\right)$ and $\left(\bar{n}_{+}, \frac{1}{2}\right)$ with $\left(n_{+}, \Delta\right)$. As one can see from Figure 2 , these results are quite accurate. 


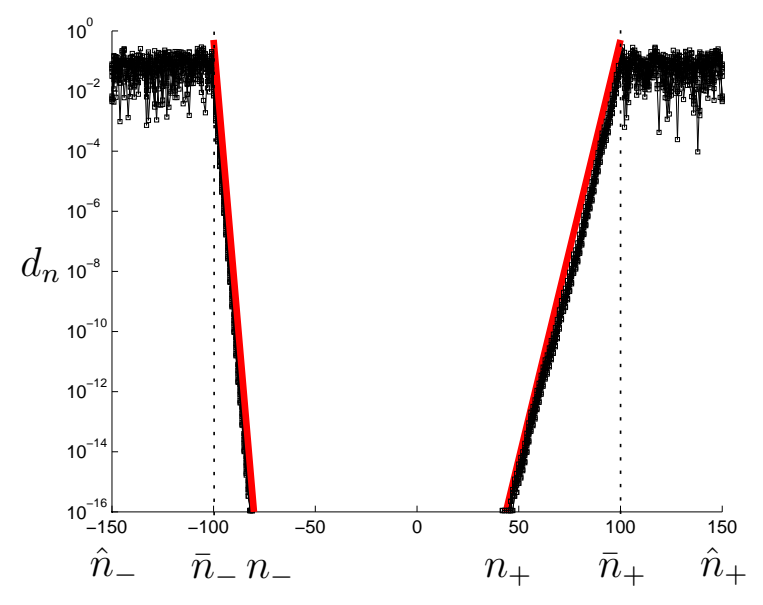

Figure 2: Difference $d_{n}=\left\|\xi_{n}-\zeta_{n}\right\|$ between two solutions of (12). $\xi_{\hat{J}}$ is computed w.r.t. the reference parameter sequence $\lambda_{\hat{J}}$, and $\zeta_{\hat{J}}$ is a solution w.r.t. a sequences $\mu_{\hat{J}}$, where $\lambda_{\hat{J}}$ and $\mu_{\hat{J}}$ coincide on $\bar{J}=\left[\bar{n}_{-}, \bar{n}_{+}\right]$. The results for 10 different $\mu_{\hat{J}}$ are shown. The red lines indicate the predicted differences.

For a randomly chosen sequence $\lambda_{\mathbb{Z}} \in I^{\mathbb{Z}}$, we illustrate the numerical approximation of a homoclinic trajectory of length $n_{-}=-20, n_{+}=20$. Compute $\bar{n}_{ \pm}$as $\bar{n}_{-}=-40, \bar{n}_{+}=74$ using (41), and solve the boundary value problem (12) on the buffer-interval $\bar{J}=\left[\bar{n}_{-}, \bar{n}_{+}\right]$, using periodic boundary conditions. Figure 3 shows the solution (left) and the accurate middle part (right).
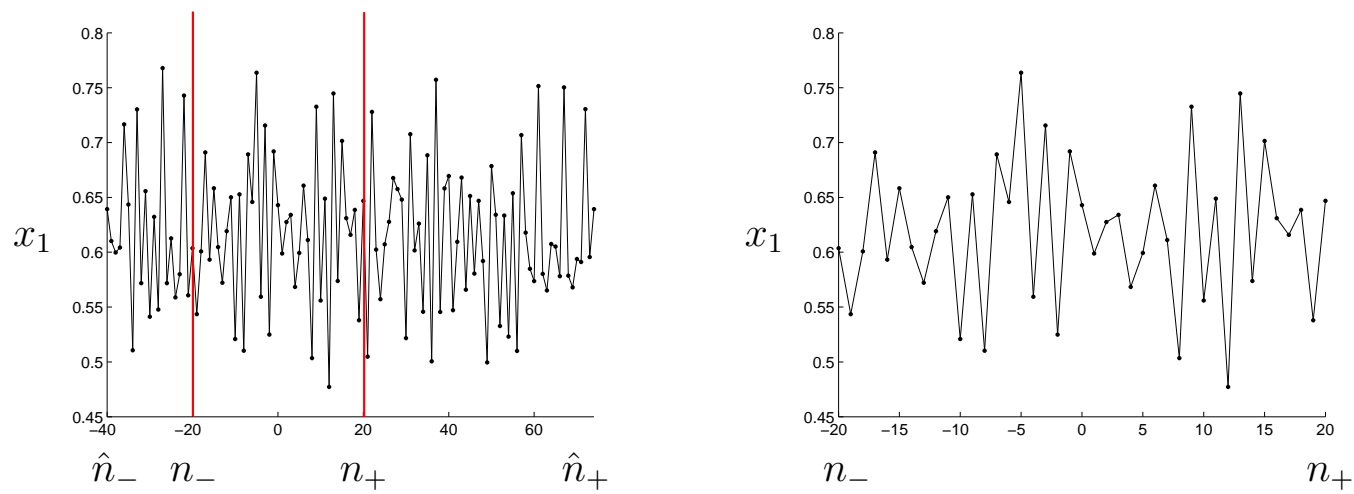

Figure 3: Bounded trajectory $\xi_{\mathbb{Z}}$ (left) and the accurate middle part (right), projected onto the $\left(n, x_{1}\right)$-plane. 


\subsection{Computation of homoclinic trajectories}

In the next step, a homoclinic orbit $y_{J}$ of the transformed system

$$
y_{n+1}=h\left(y_{n}+\xi_{n}, \lambda_{n}, b\right)-\xi_{n+1}, \quad n \in J
$$

is computed w.r.t. the fixed point 0 , see Figure 4 (left). In the right picture, the distance to the fixed point $\left\|y_{n}\right\|$ is given in a logarithmic scale, thus one can see the exponentially fast convergence of the orbit towards the fixed point 0 . Note that Newton's method is applied for computing a homoclinic orbit of (42). Due to the choice of initial points, we get a non-trivial orbit $y_{J}$, i.e. $y_{n} \neq 0$ for all $n$.

Transforming the orbit $y_{J}$ back to the original coordinates we obtain an approximation of a second trajectory $x_{J}$, where $x_{n}=y_{n}+\xi_{n}$ for $n \in J$, that is homoclinic to $\xi_{J}$. The two homoclinic trajectories $x_{J}$ (in black) and $\xi_{J}$ (in red) are shown in Figure 5 .
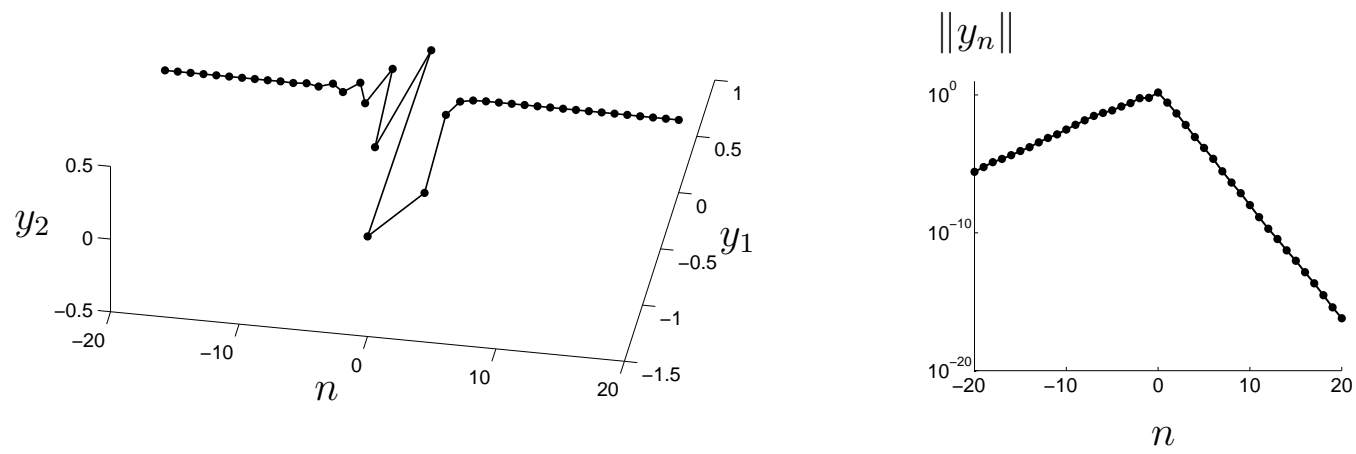

Figure 4: Homoclinic orbit of the transformed system (42) (left). The right diagram illustrates the exponentially fast convergence of the orbit towards the fixed point 0 .

\subsection{A predator-prey model}

Consider the predator-prey model

$$
\left(\begin{array}{l}
x_{n+1} \\
y_{n+1}
\end{array}\right)=G\left(\left(\begin{array}{l}
x_{n} \\
y_{n}
\end{array}\right), K\right)=\left(\begin{array}{c}
x_{n} \exp \left(a\left(1-\frac{x_{n}}{K}\right)-b y_{n}\right) \\
c x_{n}\left(1-\exp \left(-b y_{n}\right)\right)
\end{array}\right), \quad n \in \mathbb{Z},
$$

where $x_{n}\left(y_{n}\right)$ describes the number of prey (predators) at time $n$, cf. Beddington et al. (1975), Murray (2002). The parameter $K$, which we choose from an interval 

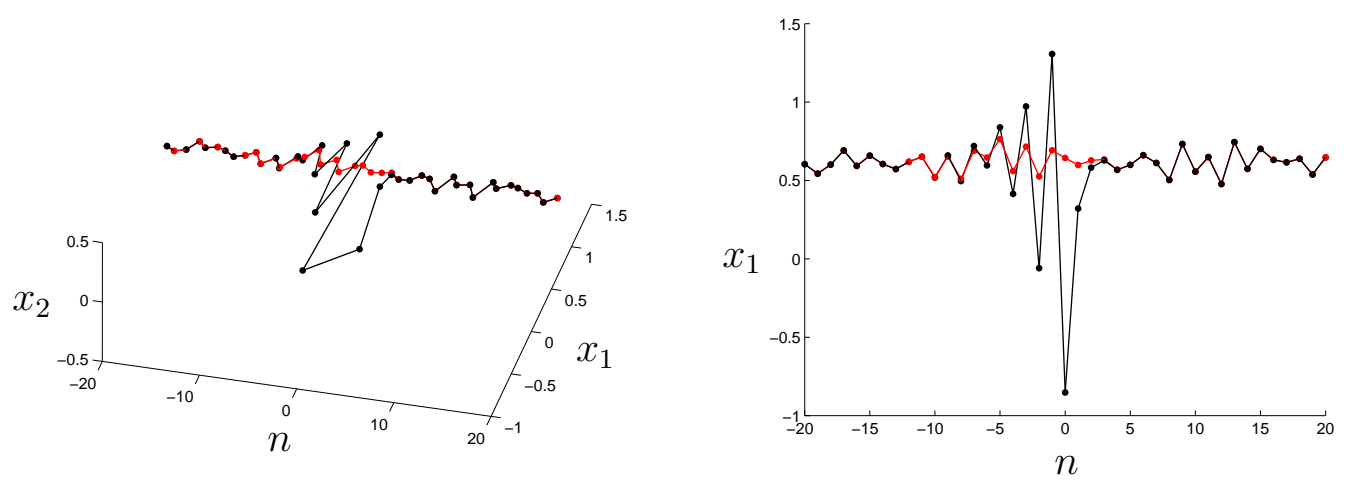

Figure 5: Two homoclinic trajectories $x_{J}$ (in black) and $\xi_{J}$ (in red). In the right figure, a projection onto the $\left(n, x_{1}\right)$ plane is given.

around 10, models the carrying capacity of the environment. More precisely, we take a sequence $r_{\mathbb{Z}}, r_{n} \in[0,1]$ for $n \in \mathbb{Z}$ at random, and define $K_{n}=10+v\left(\frac{1}{2}-r_{n}\right)$, where $v$ is the amplitude of the noise. This means that the carrying capacity fluctuates in time in the interval $\left[10-\frac{1}{2} v, 10+\frac{1}{2} v\right]$. The remaining parameters are fixed: $a=7, c=2$, and $b=0.2$.

In Figure 6 approximations of homoclinic trajectories of $G\left(\cdot, K_{n}\right)$ for the amplitudes $v \in\left\{0, \frac{1}{3}, \frac{2}{3}, 1\right\}$ are shown.

Note that in case $v=0$, the two homoclinic trajectories are a fixed point and a homoclinic orbit w.r.t. this fixed point.

For numerical computations in Figure 6 , the sequence $r_{\mathbb{Z}}$ is fixed while the amplitude varies. In this case, homoclinic trajectories depend continuously on the amplitude $v$.
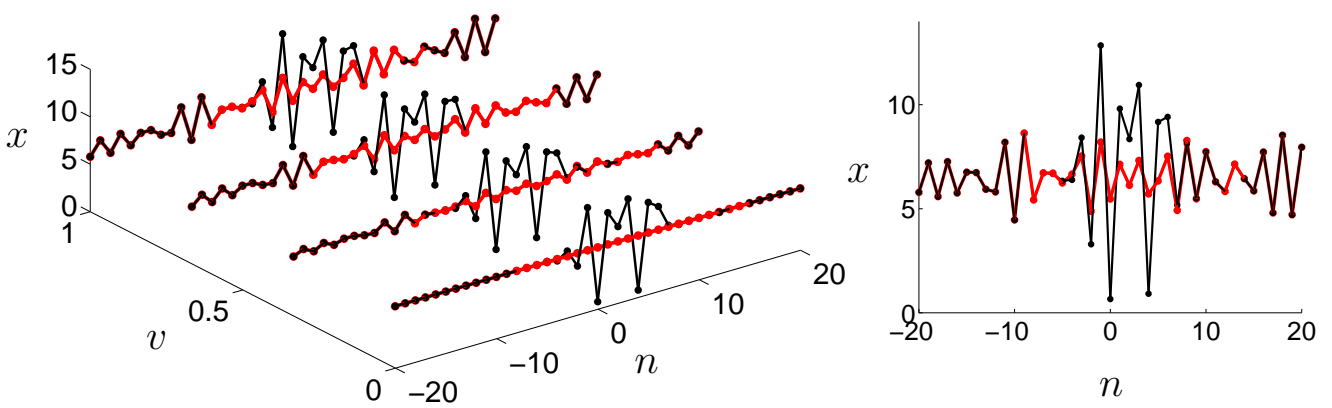

Figure 6: Numerical approximation of homoclinic trajectories on the interval $[-20,20]$ for different amplitudes of the noise $v \in\left\{0, \frac{1}{3}, \frac{2}{3}, 1\right\}$ (left) and for $v=1$ (right). 


\section{Appendix A Exponential dichotomy}

In this appendix, we collect some well known results on exponential dichotomies from Palmer (1988).

Denote by $\Phi$ the solution operator of the linear difference equation

$$
u_{n+1}=A_{n} u_{n}, \quad n \in \mathbb{Z},
$$

which is defined as

$$
\Phi(n, m):=\left\{\begin{array}{cl}
A_{n-1} \ldots A_{m}, & \text { for } n>m, \\
I, & \text { for } n=m, \\
A_{n}^{-1} \ldots A_{m-1}^{-1}, & \text { for } n<m .
\end{array}\right.
$$

Definition 13 The linear difference equation (43) with invertible matrices $A_{n} \in$ $\mathbb{R}^{k, k}$ possesses an exponential dichotomy with data $\left(K, \alpha, P_{n}^{s}, P_{n}^{u}\right)$ on $J \subset \mathbb{Z}$, if there exist two families of projectors $P_{n}^{s}$ and $P_{n}^{u}=I-P_{n}^{s}$ and constants $K, \alpha>0$, such that the following statements hold:

$$
\begin{gathered}
P_{n}^{s} \Phi(n, m)=\Phi(n, m) P_{m}^{s} \quad \forall n, m \in J, \\
\left\|\Phi(n, m) P_{m}^{s}\right\| \leq K e^{-\alpha(n-m)} \quad \forall n \geq m, n, m \in J . \\
\left\|\Phi(m, n) P_{n}^{u}\right\| \leq K \mathrm{e}^{-\alpha(n-m)} \quad
\end{gathered}
$$

We introduce an important perturbation result for exponential dichotomies, frequently named as Roughness-Theorem, cf. (Palmer 1988, Proposition 2.10).

Theorem 14 Assume that the difference equation

$$
u_{n+1}=A_{n} u_{n}, \quad A_{n} \in \mathbb{R}^{k, k} \text { invertible, } \quad\left\|A_{n}^{-1}\right\| \leq M \forall n \in J
$$

with an interval $J \subseteq \mathbb{Z}$, possesses an exponential dichotomy with data $\left(K, \alpha, P_{n}^{s}, P_{n}^{u}\right)$. Suppose $0<\delta<\alpha$ and $B_{n} \in \mathbb{R}^{k, k}$ satisfies $\left\|B_{n}\right\| \leq \beta$ for all $n \in J$, where

$$
\begin{aligned}
\beta & <M^{-1}, \\
2 K\left(1+\mathrm{e}^{-\alpha}\right)\left(1-\mathrm{e}^{-\alpha}\right)^{-1} \beta & \leq 1, \\
2 K \mathrm{e}^{\alpha}\left(\mathrm{e}^{-\delta}+1\right)\left(\mathrm{e}^{\delta}-1\right)^{-1} \beta & \leq 1 .
\end{aligned}
$$

Then $A_{n}+B_{n}$ is invertible and the perturbed difference equation

$$
u_{n+1}=\left(A_{n}+B_{n}\right) u_{n}
$$

possesses an exponential dichotomy on $J$ with data $\left(2 K\left(1+\mathrm{e}^{\delta}\right)\left(1-\mathrm{e}^{-\delta}\right)^{-1}, \alpha-\delta\right.$, $\left.Q_{n}^{s}, Q_{n}^{u}\right)$, where $\operatorname{rank}\left(Q_{n}^{s}\right)=\operatorname{rank}\left(P_{n}^{s}\right)$ and

$$
\left\|P_{n}^{s}-Q_{n}^{s}\right\| \leq 2 K^{2} \frac{1+\mathrm{e}^{-\alpha}}{1-\mathrm{e}^{-\alpha}} \sup _{m \in J}\left\|B_{m}\right\| \quad \text { for all } n \in J \text {. }
$$




\section{Appendix B A Lipschitz inverse mapping theo- rem}

We apply a quantitative version of the Lipschitz inverse mapping theorem, cf. Irwin (2001), for proving our approximation theorem.

Lemma 15 Assume $Y$ and $Z$ are Banach spaces, $F \in C^{1}(Y, Z)$ and $F^{\prime}\left(y_{0}\right)$ is for $y_{0} \in Y$ a homeomorphism. Let $\kappa, \sigma, \delta>0$ be three constants, such that the following estimates hold:

$$
\begin{aligned}
\left\|F^{\prime}(y)-F^{\prime}\left(y_{0}\right)\right\| & \leq \kappa<\sigma \leq \frac{1}{\left\|F^{\prime}\left(y_{0}\right)^{-1}\right\|} \quad \forall y \in B_{\delta}\left(y_{0}\right), \\
\left\|F\left(y_{0}\right)\right\| & \leq(\sigma-\kappa) \delta .
\end{aligned}
$$

Then $F$ has a unique zero $\bar{y} \in B_{\delta}\left(y_{0}\right)$ and the following inequalities are satisfied

$$
\begin{aligned}
\left\|F^{\prime}(y)^{-1}\right\| & \leq \frac{1}{\sigma-\kappa} \quad \forall y \in B_{\delta}\left(y_{0}\right), \\
\left\|y_{1}-y_{2}\right\| & \leq \frac{1}{\sigma-\kappa}\left\|F\left(y_{1}\right)-F\left(y_{2}\right)\right\| \quad \forall y_{1}, y_{2} \in B_{\delta}\left(y_{0}\right) .
\end{aligned}
$$

\section{Acknowledgement}

The author wishes to thank Wolf-Jürgen Beyn for stimulating discussions about this paper. The author also thanks an anonymous referee for several helpful suggestions.

\section{References}

Aulbach, B. \& Wanner, T. (2003), 'Invariant foliations and decoupling of nonautonomous difference equations', J. Difference Equ. Appl. 9(5), 459-472.

Beddington, J. R., Free, C. A. \& Lawton, J. H. (1975), 'Dynamic complexity in predator-prey models framed in difference equations', Nature 255(5503), 58-60.

Beyn, W.-J. (1990), 'The numerical computation of connecting orbits in dynamical systems.', IMA J. Numer. Anal. 10, 379-405.

Beyn, W.-J. \& Hüls, T. (2004), 'Error estimates for approximating non-hyperbolic heteroclinic orbits of maps', Numer. Math. 99(2), 289-323.

Beyn, W.-J., Hüls, T., Kleinkauf, J.-M. \& Zou, Y. (2004), 'Numerical analysis of degenerate connecting orbits for maps', Internat. J. Bifur. Chaos Appl. Sci. Engrg. 14(10), 3385-3407. 
Beyn, W.-J., Hüls, T. \& Samtenschnieder, M.-C. (2008), 'On $r$-periodic orbits of $k$-periodic maps', J. Difference Equ. Appl. 14(8), 865-887.

Beyn, W.-J. \& Kleinkauf, J.-M. (1997), 'The numerical computation of homoclinic orbits for maps', SIAM J. Numer. Anal. 34(3), 1207-1236.

Colonius, F., Hüls, T. \& Rasmussen, M. (2009), Connecting orbits in perturbed systems, Technical Report 09-008, 09-008, Bielefeld University, CRC 701. http://www.math.uni-bielefeld.de/sfb701/cgi-bin/preprints.pl.

Coomes, B. A., Koçak, H. \& Palmer, K. J. (2005), 'Homoclinic shadowing', J. Dynam. Differential Equations 17(1), 175-215.

Coomes, B. A., Koçak, H. \& Palmer, K. J. (2007), 'Transversal connecting orbits from shadowing', Numer. Math. 106(3), 427-469.

Devaney, R. L. (1989), An Introduction to Chaotic Dynamical Systems, AddisonWesley Studies in Nonlinearity, second edn, Addison-Wesley Publishing Company Advanced Book Program, Redwood City, CA.

Dhooge, A., Govaerts, W. \& Kuznetsov, Y. A. (2003), 'MATCONT: a MATLAB package for numerical bifurcation analysis of ODEs', ACM Trans. Math. Software 29(2), 141-164.

Dieudonné, J. (1969), Foundations of modern analysis, Academic Press, New York. Enlarged and corrected printing, Pure and Applied Mathematics, Vol. 10-I.

Elaydi, S. \& Sacker, R. J. (2005a), 'Global stability of periodic orbits of nonautonomous difference equations and population biology', J. Differential Equations 208(1), 258-273.

Elaydi, S. \& Sacker, R. J. (2005b), 'Nonautonomous Beverton-Holt equations and the Cushing-Henson conjectures', J. Difference Equ. Appl. 11(4-5), 337-346.

Ghaziani, R. K., Govaerts, W., Kuznetsov, Y. A. \& Meijer, H. G. E. (2009), Numerical continuation of connecting orbits of maps in MATLAB, Technical report. To appear in J. Difference Equ. Appl.

Golub, G. H. \& Van Loan, C. F. (1996), Matrix computations, Johns Hopkins Studies in the Mathematical Sciences, third edn, Johns Hopkins University Press, Baltimore, MD.

Hale, J. K. \& Koçak, H. (1991), Dynamics and Bifurcations, Vol. 3 of Texts in Applied Mathematics, Springer-Verlag, New York.

Hirsch, M. W., Pugh, C. C. \& Shub, M. (1977), Invariant manifolds, SpringerVerlag, Berlin. Lecture Notes in Mathematics, Vol. 583. 
Hüls, T. (2005), 'Bifurcation of connecting orbits with one nonhyperbolic fixed point for maps', SIAM J. Appl. Dyn. Syst. 4(4), 985-1007 (electronic).

Hüls, T. (2006), 'Homoclinic orbits of non-autonomous maps and their approximation', J. Difference Equ. Appl. 12(11), 1103-1126.

Hüls, T. (2008), Numerical computation of dichotomy rates and projectors in discrete time, Technical Report 08-081, 08-081, Bielefeld University, CRC 701. http://www.math.uni-bielefeld.de/sfb701/cgi-bin/preprints.pl. To appear in Discrete and Continuous Dynamical Systems-Series B.

Irwin, M. C. (2001), Smooth dynamical systems, Vol. 17 of Advanced Series in Nonlinear Dynamics, World Scientific Publishing Co. Inc., River Edge, NJ. Reprint of the 1980 original, With a foreword by R. S. MacKay.

Langa, J. A., Robinson, J. C. \& Suárez, A. (2002), 'Stability, instability, and bifurcation phenomena in non-autonomous differential equations', Nonlinearity 15(3), 887-903.

Mira, C. (1987), Chaotic dynamics, World Scientific Publishing Co., Singapore. From the one-dimensional endomorphism to the two-dimensional diffeomorphism.

Murray, J. D. (2002), Mathematical biology. I, Vol. 17 of Interdisciplinary Applied Mathematics, third edn, Springer-Verlag, New York. An introduction.

Palis, J. \& Takens, F. (1993), Hyperbolicity and Sensitive Chaotic Dynamics at Homoclinic Bifurcations, Vol. 35 of Cambridge Studies in Advanced Mathematics, Cambridge University Press, Cambridge.

Palmer, K. J. (1988), Exponential dichotomies, the shadowing lemma and transversal homoclinic points, in 'Dynamics reported, Vol. 1', Teubner, Stuttgart, pp. 265306.

Pilyugin, S. Y. (1999), Shadowing in Dynamical Systems, Vol. 1706 of Lecture Notes in Mathematics, Springer-Verlag, Berlin.

Pötzsche, C. \& Siegmund, S. (2004), ' $C^{m}$-smoothness of invariant fiber bundles', Topol. Methods Nonlinear Anal. 24(1), 107-145.

Smale, S. (1967), 'Differentiable dynamical systems', Bull. Amer. Math. Soc. 73, $747-817$. 